\title{
On Singular Control Problems with State Constraints and Regime-Switching: A Viscosity Solution Approach
}

\author{
Qingshuo Song ${ }^{\mathrm{a}}$, Chao Zhu ${ }^{\mathrm{b}}$ \\ ${ }^{a}$ Department of Mathematics, City University of Hong Kong, 83 Tat Chee Avenue, Kowloon Tong, Hong Kong. \\ ${ }^{\mathrm{b}}$ Department of Mathematical Sciences, University of Wisconsin-Milwaukee, Milwaukee, WI 53201, USA.
}

\begin{abstract}
This paper investigates a singular stochastic control problem for a multi-dimensional regime-switching diffusion process confined in an unbounded domain. The objective is to maximize the total expected discounted rewards from exerting the singular control. Such a formulation stems from application areas such as optimal harvesting multiple species and optimal dividends payments schemes in random environments. With the aid of weak dynamic programming principle and an exponential transformation, we characterize the value function to be the unique constrained viscosity solution of a certain system of coupled nonlinear quasi-variational inequalities. Several examples are analyzed in details to demonstrate the main results.
\end{abstract}

Key words: Constrained viscosity solution, regime-switching diffusion, singular stochastic control, weak dynamic programming principle, quasi-variational inequality.

\section{Introduction}

This paper is concerned with a class of singular stochastic control problems with state constraints. The controlled regime-switching diffusion process $X$ and the singular control process $Z$ take values in a convex cone $S \subset \mathbb{R}^{n}$. The control problem has the state process

$$
\begin{aligned}
X(t)=x & +\int_{0}^{t} b(X(s), \alpha(s)) d s \\
& +\int_{0}^{t} \sigma(X(s), \alpha(s)) d W(s)-Z(t),
\end{aligned}
$$

where $x \in \mathbb{R}^{n}, W$ is a $d$-dimensional standard Brownian motion, $\alpha(\cdot) \in \mathcal{M}=\{1, \ldots, m\}$ is a continuous-time Markov chain that is independent of the Brownian mo-

\footnotetext{
* The research of Qingshuo Song was supported in part by the RGC of CityU 100310 and the CityU SRG 2010SRG069. The research of Chao Zhu was supported in part by the National Science Foundation under DMS-1108782 and a grant from the UWM Research Growth Initiative.

Email addresses: song.qingshuo@cityu.edu.hk (Qingshuo Song), zhu@uwm.edu (Chao Zhu).
}

tion $W$ and is generated by $Q=\left(q_{i j}\right) \in \mathbb{R}^{m \times m}$ :

$$
\begin{array}{r}
\mathbb{P}\{\alpha(t+\Delta t)=j \mid \alpha(t)=i, \alpha(s), s \leq t\} \\
= \begin{cases}q_{i j} \Delta t+o(\Delta t), & \text { if } j \neq i \\
1+q_{i i} \Delta t+o(\Delta t), & \text { if } j=i,\end{cases}
\end{array}
$$

where $q_{i j} \geq 0$ for $i, j=1, \ldots, m$ with $j \neq i$ and $q_{i i}=$ $-\sum_{j \neq i} q_{i j}<0$ for each $i=1, \ldots, m$.

The control process $Z=\left(Z_{1}, \ldots, Z_{n}\right)^{\prime}$ is further required to be an $n$-dimensional adapted, nondecreasing, and càdlàg stochastic process, which belongs to a control space $\mathcal{A}_{x, \alpha}$ to be defined later. The objective is to maximize the total discounted reward

$$
\begin{aligned}
& J(x, \alpha, Z) \\
& \quad:=\mathbb{E}\left[\int_{0}^{\infty} e^{-r s} f\left(X^{x, \alpha}(s-), \alpha(s-)\right) \cdot d Z(s)\right],
\end{aligned}
$$

where $r>0, f: S \times \mathcal{M} \mapsto \mathbb{R}^{n}$ with $f_{i}$ representing the state- and regime-dependent instantaneous marginal yields accrued from exerting the singular control $Z_{i}(t)$.

Such singular control problems (in various different settings) have been extensively studied in the literature. A partial list includes the monotone follower problems (Karatzas and Shreve (1984)), optimal harvesting problems (Alvarez and Shepp (1998), Song et al. (2011)), 
portfolio selection management with transaction costs (Øksendal and Sulem (2002)), optimal partially reversible investment problem (Guo and Pham (2005)), etc. See also Haussmann and Suo (1995a,b) for a general singular stochastic control problem for a multidimensional Itô diffusion on a fixed time horizon, in which the existence of the optimal control and the characterization of the value function as the unique viscosity solution of a Hamilton-Jacobi-Bellman equation are established. Singular control problems with state constraints have drawn considerable interests in recent years; see, for example, Atar and Budhiraja (2006), Atar et al. (2007), Zariphopoulou (1992), among others.

Note that most, if not all, of the aforementioned literature on singular stochastic controls deal with Itô (jump) diffusions. One exception is our recent work Song et al. (2011), which studies an optimal harvesting problem of a single species living in random environments. Due to their capability of modeling complex systems with uncertainty, regime-switching models have drawn considerable attention from both researchers and practitioners in recent decades in a wide range of applications. Some of such examples can be found in mathematical finance (Zhang (2001)), ecosystem modeling (Zhu and Yin (2009)), stochastic manufacturing systems (Sethi and Zhang (1994)), risk management (Elliott and Siu (2010), Zhu (2011)), to name just a few. In these systems, both continuous dynamics and discrete events coexist. In particular, the systems often display qualitative structural changes. Regime-switching models turn out to be quite versatile in capturing these inherent randomness. We refer to Mao and Yuan (2006) and Yin and Zhu (2010) for in-depth investigations of regime-switching diffusions.

This paper aims to investigate the singular control problem (1.3) in the setting of multi-dimensional regimeswitching diffusion with state constraints. In particular, motivated by several simple yet nontrivial examples, we derive a strong comparison result from which we establish the existence and uniqueness of the constrained viscosity solution to (2.2).

Compared with the classical work on viscosity solution such as Crandall et al. (1992) and others, the novelty and contribution of this work can be summarized as follows. In lieu of a single differential equation studied in the literature, this work deals with a coupled system of nonlinear second-order differential equations with gradient constraints. This is due to the presence of random environments or regime switching. This feature at one hand makes our model more appealing in real-world applications since it can naturally capture the qualitative structural changes of the systems; on the other hand, it adds much difficulty in the analysis. In particular, the function $F$ defined in (2.3) is not proper in the sense of the User's Guide Crandall et al. (1992). Note that the properness was an essential assumption in the proof of the strong comparison result in Crandall et al. (1992).
Here we need to carefully handle the coupling effect; see the proof of Theorem 11 for more details. Another noteworthy feature of this work is that we introduce an exponential transformation which allows us to handle both the gradient constraints as well as the polynomial growth condition on an unbounded domain for the solution of the quasi-variational inequalities (QVIs) (2.2).

The paper Ishii (1989) also contains comparison results (Theorems 7.1 and 7.3) for viscosity solutions of fully nonlinear second order PDEs $F\left(x, u, D u, D^{2} u\right)=0$ in unbounded domain $\Omega$. In deriving those results, in addition to the growth conditions of the solutions and some other conditions, the function $F(x, r, p, X)$ is assumed to be nondecreasing the $r$ variable. As we mentioned earlier, our function $F$ defined in (2.3) does not satisfy this assumption. Consequently we can not apply the techniques in Ishii (1989) directly. Moreover, we need to take care of the gradient constraints in (2.2).

In addition, there are a few key differences between this paper and our previous work Song et al. (2011). By exploiting the advantage of dealing with a one-dimensional problem, Song et al. (2011) first derived a sufficient condition under which the value function is continuous, which, in turn, led to the existence proof of showing that it is $a$ viscosity solution of the corresponding QVIs. In this paper, we use the weak dynamic programming principle to show directly that the value function is a constrained viscosity solution to the corresponding coupled system of QVIs (2.2). Moreover, with the aid of an exponential transformation, we establish a strong comparison theorem and hence the uniqueness part of constrained viscosity solution to (2.2). In this way, we obtain a complete characterization of the value function (2.1), i.e., it is the unique constrained viscosity solution to (2.2).

The rest of the paper is arranged as follows. Section 2 presents the precise formulation of the problem, followed by some preliminary results in Section 3 . We recall the notion of constrained viscosity solution in Section 4 , followed by several examples for illustration. Further, in Section 4, we establish the existence by showing that the value function $V$ defined in (2.1) is a constrained viscosity solution of (2.2). The strong comparison result is arranged in Section 5. The paper is concluded with conclusions and remarks in Section 6.

To facilitate later presentation, we introduce some notations that will be used often in later sections. For a càdlàg (right continuous with left limits) function $\xi$ : $[0, \infty) \mapsto \mathbb{R}^{n}$, we write $\Delta \xi(t)=\xi(t)-\xi(t-)$ for $t>$ 0 . As a convention, we set $\Delta \xi(0)=\xi(0)$. Throughout the paper, we use $x^{\prime} y$ or $x \cdot y$ interchangablly to denote the inner product of vectors $x$ and $y$. For any vectors $x, y \in \mathbb{R}^{n}, x \leq y$ means $x_{i} \leq y_{i}$ for every $i=1, \ldots, n$. The space of $n \times n$ symmetric matrices is denoted by $\mathcal{S}_{n}$ and the family of positive definite symmetric matrices is denoted by $\mathcal{S}_{n}^{+}$. If $\phi: \mathbb{R}^{n} \rightarrow \mathbb{R}$ is suf- 
ficiently smooth, then $D_{x_{i}} \phi=\frac{\partial \phi}{\partial x_{i}}, D_{x_{i} x_{j}} \phi=\frac{\partial^{2} \phi}{\partial x_{i} \partial x_{j}}$, and $D \phi=\left(D_{x_{1}} \phi, \ldots, D_{x_{n}} \phi\right)^{\prime}$ is the gradient of $\phi$ while $D^{2} \phi=\left(D_{x_{i} x_{j}} \phi\right)$ denotes the Heissian of $\phi$. For any real-valued function $f$, we use $f_{*}$ and $f^{*}$ to denote the lower- and upper-semicontinuous envelopes of $f$, respectively. That is, $f_{*}(x):=\liminf _{y \rightarrow x} f(y)$ and $f^{*}(x):=$ $\limsup _{y \rightarrow x} f(y)$. If $f: \mathbb{R}^{n} \times \mathcal{M} \mapsto \mathbb{R}$, with a slight abuse of notation, we define $f_{*}(x, i):=\liminf _{y \rightarrow x} f(y, i)$ and $f^{*}(x, i):=\limsup _{y \rightarrow x} f(y, i)$ for all $(x, i) \in \mathbb{R} \times \mathcal{M}$.

If $B$ is a set, we use $B^{o}$ and $I_{B}$ to denote the interior and indicator function of $B$, respectively. Throughout the paper, we adopt the conventions that $\sup \emptyset=-\infty$ and $\inf \emptyset=+\infty$.

\section{Formulation}

Let $\zeta$ be a regime-switching diffusion process given by (1.1) with $Z \equiv 0$. Throughout the paper, we assume that the coefficients $b$ and $\sigma$ and the generator $Q$ are such that for any initial condition $(x, \alpha) \in \mathbb{R}^{n} \times \mathcal{M}$, the solution $\zeta^{x, \alpha}$ to (1.1) (with $Z \equiv 0$ ) exists and is pathwise unique. Sufficient condition for existence and uniqueness for stochastic differential equations with regime switching can be found in, for example, Mao and Yuan (2006), Yin and Zhu (2010).

Let $\mathcal{A}_{x, \alpha}$ denote the collection of all admissible controls with initial conditions $(x, \alpha)$, where $Z \in \mathcal{A}_{x, \alpha}$ satisfies

(i) for each $i=1, \ldots, n, Z_{i}(t)$ is nonnegative, càdlàg and nondecreasing with respect to $t$,

(ii) $X(t) \in \bar{S}$ for all $t \geq 0$, and

(iii) $Z(t)$ is adapted to $\mathfrak{F}_{t}:=\sigma\{W(s), \alpha(s), 0 \leq s \leq t\}$, where $\mathfrak{F}_{0}$ contains all $\mathbb{P}$-null sets. Moreover,

$$
\mathbb{E}\left[\int_{0}^{\infty} e^{-r s} d|Z|(s)\right]<\infty .
$$

Note that the state constraint is specified in condition (ii) above. Such a constraint and the consideration of the nonnegative and nondecreasing control processes $Z_{i}, i=$ $1, \ldots, n$ are motivated by many applications such as mathematical finance (Øksendal and Sulem (2002)), optimal harvesting problems (Alvarez and Shepp (1998), Song et al. (2011)), among others. We also assume the income rate $f_{i}$ is continuous and non-increasing with respect to $x$ in the sense that $f_{i}(x, \alpha) \geq f_{i}(y, \alpha)$ for each $\alpha \in \mathcal{M}$ if $x \leq y$, where $x=\left(x_{1}, \ldots, x_{n}\right)^{\prime}$ and $y=$ $\left(y_{1}, \ldots, y_{n}\right)^{\prime}$ satisfy $x_{j} \leq y_{j}$ for each $j=1, \ldots, n$. Moreover, we assume $0<f_{i}(0, \alpha)<\infty$ for each $i=1, \ldots, n$ and $\alpha \in \mathcal{M}$. Such assumptions on $f$ are motivated by the optimal harvesting problems considered in Alvarez (2000) and Song et al. (2011). The goal is to maximize the expected total discounted payoff $J$ defined in (1.3) and find an optimal control $Z^{*}$ :

$$
V(x, \alpha)=J\left(x, \alpha, Z^{*}\right):=\sup _{Z \in \mathcal{A}_{x, \alpha}} J(x, \alpha, Z) .
$$

In order to work with a well-formulated maximization problem, we assume throughout the paper that $V(x, \alpha)<\infty$ for all $(x, \alpha) \in \mathbb{R}_{+}^{n} \times \mathcal{M}$; see Remark 12 for a sufficient condition.

As usual, we shall rely on the dynamic programming principle (DPP) to deduce the behavior of the value function $V(x, \alpha)$ for every $(x, \alpha) \in S \times \mathcal{M}$ and stopping time $\eta$. A heuristic argument using the DPP yields that $\mathbf{V}(x)=(V(x, 1), \ldots, V(x, m))^{\prime} \in \mathbb{R}^{m}$ satisfies a coupled system of quasi-variational inequalities (QVIs):

$$
\begin{array}{r}
\min \left\{F_{\alpha}\left(x, \mathbf{V}(x), D V(x, \alpha), D^{2} V(x, \alpha)\right),\right. \\
\left.\quad \min \left\{D_{x_{i}} V(x, \alpha)-f_{i}(x, \alpha)\right\}\right\}=0,
\end{array}
$$

for all $x \in S$ and each $\alpha=1, \ldots, m$. Here and throughout, for all $(x, \alpha, \xi, p, A) \in \mathbb{R}^{n} \times \mathcal{M} \times \mathbb{R}^{m} \times \mathbb{R}^{n} \times \mathcal{S}_{n}$,

$$
\begin{aligned}
& F_{\alpha}(x, \xi, p, A)=F(x, \alpha, \xi, p, A) \\
& :=r \xi_{\alpha}-\frac{1}{2} \operatorname{tr}\left(\sigma \sigma^{\prime}(x, \alpha) A\right)-b(x, \alpha) \cdot p-\sum_{j=1}^{m} q_{\alpha j} \xi_{j} .
\end{aligned}
$$

However, without a priori result on the continuity of the value function, a rigorous proof of DPP is nontrivial. Due to the state constraint as well as the generality of the set up of the problem, it seems not easy to obtain the continuity of the value function $V$. To overcome this difficulty, we will instead invoke the weak DPP (Bouchard and Touzi (2011)); see Proposition 2 for the precise statement. Also, the value function $V$ is not necessarily sufficiently smooth to take first and second order partial derivatives. Therefore we aim to show that $V$ satisfies QVIs in the weak sense using the notion of viscosity solution. Indeed, Theorem 13 asserts that the value function $V$ is the unique viscosity solution to QVIs.

As we indicated earlier, (2.2) is a coupled system of QVIs. Moreover, thanks to the term $\sum_{j=1}^{m} q_{\alpha j} \xi_{j}$ with $Q=\left(q_{i j}\right)$ defined in (1.2), for each $\alpha \in \mathcal{M}, F_{\alpha}$ is not proper with respect to the variable $\xi$ in the sense of equations (0.1) or (0.2) in the User's Guide Crandall et al. (1992). Note that the properness assumption (and in particular equation (3.13) in Crandall et al. (1992)) enabled them to derive the strong comparison result and hence the uniqueness of the viscosity solution. Here for our analysis, special care has to be given to handle the fact that $F_{\alpha}$ is not proper due to the coupling term. Also, instead of working on a bounded domain, we are dealing with unbounded domain $S$. These features make our analysis much more involved than the classical comparison result in Crandall et al. (1992). 


\section{Some Preliminary Results}

We present some preliminary results in this section. The first one provides a sufficient condition for the assumption that $\mathcal{A}_{x, \alpha} \neq \emptyset$ for all $(x, \alpha) \in S \times \mathcal{M}$.

Proposition 1 Assume there exists a function $\Psi: S \times$ $\mathcal{M} \mapsto \mathbb{R}_{+}$satisfying

(i) for each $i=1, \ldots, n$ and $\alpha \in \mathcal{M}, \lim _{|x| \rightarrow \infty} \Psi(x, \alpha)$ $=\infty$, and $\lim _{x_{i} \downarrow 0} \Psi(x, \alpha)=\infty$,

(ii) $\Psi(\cdot, \alpha) \in C^{2}$ for each $\alpha \in \mathcal{M}$ and $\mathcal{L} \Psi(x, \alpha) \leq K$ for all $(x, \alpha) \in S \times \mathcal{M}$, where $K>0$ is a constant and

$$
\begin{aligned}
\mathcal{L} \Psi(x, \alpha)=b(x, \alpha) & \cdot D \Psi(x, \alpha)+\sum_{j=1}^{m} q_{\alpha j} \Psi(x, j) \\
+ & \frac{1}{2} \operatorname{tr}\left(\sigma \sigma^{\prime}(x, \alpha) D^{2} \Psi(x, \alpha)\right) .
\end{aligned}
$$

Then we have

$$
\mathbb{P}\left\{\zeta^{x, \alpha}(t) \in S \text { for all } t \geq 0\right\}=1, \forall(x, \alpha) \in S \times \mathcal{M}
$$

Consequently $\mathcal{A}_{x, \alpha} \neq \emptyset$ for all $(x, \alpha) \in S \times \mathcal{M}$.

Proof. It suffices to prove (3.1), from which we have $Z \equiv 0 \in \mathcal{A}_{x, \alpha}$ for any $(x, \alpha) \in S \times \mathcal{M}$. To this end, we consider $(x, \alpha) \in S \times \mathcal{M}$ and define for $k \geq k_{0}$

$$
\begin{array}{r}
\tau_{k}:=\inf \left\{t \geq 0:\left|\zeta^{x, \alpha}(t)\right| \geq k \text { or } \zeta_{i}^{x, \alpha}(t) \leq \frac{1}{k}\right. \\
\text { for some } i=1, \ldots, n\}
\end{array}
$$

where $k_{0} \in \mathbb{N}$ is such that $|x|<k_{0}$ and $\min \left\{x_{i}, i=\right.$ $1, \ldots, n\}>\frac{1}{k_{0}}$. Note that $\left\{\tau_{k}\right\}$ is nondecreasing. Denote $\tau_{\infty}:=\lim _{k \rightarrow \infty} \tau_{k}$. Now (3.1) will follow if we can show that $\tau_{\infty}=\infty$ a.s. Suppose on the contrary that there exist some $T>0$ and $\delta>$ such that $\mathbb{P}\left\{\tau_{\infty} \leq T\right\} \geq \delta$. Then there is a $k_{1} \in \mathbb{N}$ such that

$$
\mathbb{P}\left\{\tau_{k} \leq T\right\} \geq \delta, \quad \forall k \geq k_{1}>k_{0}
$$

Applying generalized Itô's formula to the function $\Psi$ and using condition (ii), we obtain that for any $k \geq k_{1}$,

$$
\begin{aligned}
\mathbb{E} & {\left[\Psi\left(\zeta\left(\tau_{k} \wedge T\right), \alpha\left(\tau_{k} \wedge T\right)\right]\right.} \\
& =\Psi(x, \alpha)+\mathbb{E}\left[\int_{0}^{\tau_{k} \wedge T} \mathcal{L} \Psi(\zeta(s), \alpha(s)) d s\right] \\
& \leq \Psi(x, \alpha)+K T .
\end{aligned}
$$

Since $\Psi \geq 0$, it follows from condition (i) and (3.2) that

$$
\begin{aligned}
\infty>\Psi(x, \alpha)+K T & \geq \mathbb{E}\left[\Psi\left(\zeta\left(\tau_{k}\right), \alpha\left(\tau_{k}\right) I_{\left\{\tau_{k} \leq T\right\}}\right]\right. \\
& \geq \Psi_{k} \mathbb{P}\left\{\tau_{k} \leq T\right\} \rightarrow \infty, \text { as } k \rightarrow \infty,
\end{aligned}
$$

where $\Psi_{k}:=\inf \left\{\Psi(x, j),|x|=k\right.$ or $x_{i}=\frac{1}{k}$ for some $i=1, \ldots, n$, and $j \in \mathcal{M}\}$. This is a contradiction and hence $\tau_{k} \rightarrow \infty$ with probability 1 as $k \rightarrow \infty$.

Next we make a couple of observations. For any $(x, \alpha) \in$ $\bar{S} \times \mathcal{M}, \mathcal{A}_{x, \alpha} \neq \emptyset$ if and only if $0 \equiv Z \in \mathcal{A}_{x, \alpha}$. Furthermore, it is easy to see $0 \equiv Z \in \mathcal{A}_{x, \alpha}$ if and only if $\mathbb{P}_{x, \alpha}\{\vartheta<\infty\}=0$, where $\vartheta:=\inf \left\{t \geq 0: \zeta^{x, \alpha}(t) \in \bar{S}^{c}\right\}$ is the first entrance time into $\bar{S}^{c}$.

For ease of presentation, similar to the classification of boundary points for one-dimensional regular diffusion processes, we say that $x \in \partial S$ is an irregular point if $\mathbb{P}_{x, \alpha}\{\vartheta<\infty\}=0$ for each $\alpha \in \mathcal{M}$, where $\vartheta$ is the first entrance time of $\zeta^{x, \alpha}$ into $\bar{S}^{c}$. Note that an entrance point is an irregular boundary point. As in (Karlin and Taylor, 1981, Chapter 15), an entrance point $x \in \partial S$ can not be reached from the interior and is such that the process, starting from $x$, enters the interior of $S$ "in no time," i.e., $\mathbb{P}\{\theta=0\}=1$, where $\theta:=\inf \left\{t \geq 0: \zeta^{x, \alpha}(t) \in S\right\}$. Likewise, an exit-trap-absorbing point is an irregular point. Again, as in (Karlin and Taylor, 1981, Chapter $15)$, we say that $x \in \partial S$ is an exit-trap-absorbing point if once the process $\zeta$ reaches $x$, it remains in $\partial S$ forever.

The next proposition can be established using similar arguments as those in Bouchard and Touzi (2011).

Proposition 2 Fix $(x, \alpha) \in \mathbb{R}_{+}^{n} \times \mathcal{M}$. Then for any stopping time $\tau$, we have

$$
\begin{array}{r}
V(x, \alpha) \leq \sup _{Z \in \mathcal{A}_{x, \alpha}} \mathbb{E}\left[e^{-r \tau} V^{*}\left(X^{x, \alpha}(\tau), \alpha(\tau)\right)\right. \\
+\int_{0}^{\tau} e^{-r s} f\left(X^{x, \alpha}(s-), \alpha(s-) \cdot d Z(s)\right] \\
V(x, \alpha) \geq \sup _{Z \in \mathcal{A}_{x, \alpha}} \mathbb{E}\left[e^{-r \tau} \varphi\left(X^{x, \alpha}(\tau), \alpha(\tau)\right)\right. \\
+\int_{0}^{\tau} e^{-r s} f\left(X^{x, \alpha}(s-), \alpha(s-) \cdot d Z(s)\right]
\end{array}
$$

for all upper-semicontinuous functions $\varphi$ such that $V \geq$ $\varphi$ on $\mathbb{R}_{+}^{n} \times \mathcal{M}$

In addition, we will need the following proposition in the proof of Theorem 8 .

Proposition 3 For each $\alpha \in \mathcal{M}$ and any $x, y \in S$ with $y \leq x$, we have

$$
\begin{gathered}
V(x, \alpha) \geq f(x, \alpha) \cdot(x-y)+V(y, \alpha), \\
V^{*}(x, \alpha) \geq f(x, \alpha) \cdot(x-y)+V^{*}(y, \alpha) .
\end{gathered}
$$

Proof. Equation (3.5) can be established using exactly the same arguments as those in Song et al. (2011), while (3.6) follows from (3.5) directly. 


\section{Viscosity Solution: Existence}

This section is devoted to the properties of the value function $V$. In particular, we aim to characterize $V$ as a viscosity solution to the QVIs (2.2). Let's first recall the notion of viscosity solution.

Definition 4 A function $\mathbf{u}(x)=(u(x, 1), \ldots, u(x, m))^{\prime}$ is said to be a viscosity subsolution of (2.2) on $\bar{S} \times \mathcal{M}$, if for any $\left(x_{0}, \alpha_{0}\right) \in \bar{S} \times \mathcal{M}$ and functions $\varphi(\cdot, \alpha) \in C^{2}(S), \alpha \in \mathcal{M}$ satisfying_ $\left(u^{*}-\varphi\right)(x, \alpha) \leq$ $\left(u^{*}-\varphi\right)\left(x_{0}, \alpha_{0}\right)=0$ for all $(x, \alpha) \in \bar{S} \times \mathcal{M}$, we have

$$
\begin{array}{r}
\min \left\{F_{\alpha_{0}}\left(x_{0}, \mathbf{u}^{*}\left(x_{0}\right), D \varphi\left(x_{0}, \alpha_{0}\right), D^{2} \varphi\left(x_{0}, \alpha_{0}\right)\right),\right. \\
\left.\min _{i=1, \ldots, n}\left\{D_{x_{i}} \varphi\left(x_{0}, \alpha_{0}\right)-f_{i}\left(x_{0}, \alpha_{0}\right)\right\}\right\} \leq 0 .
\end{array}
$$

Similarly, a function $\mathbf{u}(x)=(u(x, 1), \ldots, u(x, m))^{\prime}$ is said to be a viscosity supersolution of $(2.2)$ in $S \times \mathcal{M}$, if for any $\left(x_{0}, \alpha_{0}\right) \in S \times \mathcal{M}$ and functions $\varphi(\cdot, \alpha) \in C^{2}(S), \alpha \in$ $\mathcal{M}$ satisfying $\left(u_{*}-\varphi\right)(x, \alpha) \geq\left(u_{*}-\varphi\right)\left(x_{0}, \alpha_{0}\right)=0$ for all $(x, \alpha) \in S \times \mathcal{M}$, we have

$$
\begin{array}{r}
\min \left\{F_{\alpha_{0}}\left(x_{0}, \mathbf{u}_{*}\left(x_{0}\right), D \varphi\left(x_{0}, \alpha_{0}\right), D^{2} \varphi\left(x_{0}, \alpha_{0}\right)\right),\right. \\
\left.\min _{i=1, \ldots, n}\left\{D_{x_{i}} \varphi\left(x_{0}, \alpha_{0}\right)-f_{i}\left(x_{0}, \alpha_{0}\right)\right\}\right\} \geq 0 .
\end{array}
$$

The function $u$ is said to be a constrained viscosity solution, if it is both a viscosity subsolution in $\bar{S} \times \mathcal{M}$ and a viscosity supersolution in $S \times \mathcal{M}$.

Before presenting the main result of this section, we shall first study several examples to illustrate Definition 4. These examples will also help us to motivate later results.

\section{Example 5 Consider the QVI}

$$
\min \left\{u(x)-u^{\prime}(x), u^{\prime}(x)-1\right\}=0, \quad x \in(0, \infty) .
$$

Using Definition 4 , we can easily verify that $u(x)=K e^{x}$ for $K \geq 1$ and $v(x)=x+1$ on $[0, \infty)$ are constrained viscosity solutions to (4.1).

The controlled process corresponding to (4.1) is $X(t)=$ $x+t-Z(t)$ for $t \geq 0$ and the objective is to maximize $J(x, Z)=\mathbb{E}_{x} \int_{0}^{\infty} e^{-t} d Z(t)$. Moreover, from the state constraint, $Z(t) \leq x+t$ for all $t \geq 0$. Then, one can easily determine the value function $V(x)=x+1$ and an optimal control policy $Z^{*}(t):=x I_{\{t=0\}}+I_{\{t>0\}} \int_{0}^{t} 1 d s$.

To conclude, the value function $V(x)=x+1$ is the unique constrained viscosity solution of $(4.1)$ on $[0, \infty)$ in the class of functions with polynomial growth rate. $\square$
Example 6 We demonstrate that the QVI

$$
\min \left\{u(x)-u^{\prime \prime}(x), u^{\prime}(x)-1\right\}=0, \quad x \in(0, \infty)
$$

has no constrained viscosity solution on $[0, \infty)$.

First, one can show that none of the function of the form $u(x)=x+c$ or $u(x)=c_{1} e^{x}+c_{2} e^{-x}$ is a constrained viscosity solution of $(4.2)$ on $[0, \infty)$, where $c, c_{1}, c_{2}$ are constants. Hence any linear combination of $x+c$ and $c_{1} e^{x}+c_{2} e^{-x}$ can not be a constrained viscosity solution of $(4.2)$ on $[0, \infty)$ either. In addition, functions of the form $u(x)=(x+c) I_{\{x>a\}}+\left(c_{1} e^{x}+c_{2} e^{-x}\right) I_{\{x \leq a\}}$ are not constrained viscosity solution of $(4.2)$ on $[0, \infty)$, where $a, c_{1}, c_{2}$ are appropriately selected constants so that $u \in$ $C^{1}([0, \infty)) \cap C^{2}([0, \infty)-\{a\})$ and solves $(4.2)$ in $(0, \infty)$.

Finally we note that for the corresponding controlled process $X(t)=x+\sqrt{2} W(t)$ and the reward functional $\mathbb{E}_{x}\left[\int_{0}^{\infty} e^{-t} d Z(t)\right], \mathcal{A}_{0}=\emptyset$. The reason is that the Brownian motion $W$, starting from 0 , changes sign infinitely many times and hence can not satisfy the state constraint in any time interval $[0, \varepsilon]$.

Example 7 We consider the system of coupled QVIs

$$
\begin{aligned}
& \min \left\{r u(x, \alpha)-\mu_{\alpha} x u^{\prime}(x, \alpha)-\frac{1}{2} \sigma_{\alpha}^{2} x^{2} u^{\prime \prime}(x, \alpha)\right. \\
& \left.-\lambda_{\alpha} u(x, \alpha)+\lambda_{\alpha} u(x, 3-\alpha), u^{\prime}(x, \alpha)-1\right\}=0,
\end{aligned}
$$

$x \in(0, \infty), \alpha \in\{1,2\}$, where for $\alpha=1,2, \mu_{\alpha}, \sigma_{\alpha}$, and $\lambda_{\alpha}>0$ are constants. Moreover, we assume $\mu_{1}, \mu_{2}$ satisfy $\mu_{1}<r<\mu_{2} \leq \frac{r \lambda_{1}+\left(r-\mu_{1}\right)\left(r+\lambda_{2}\right)}{r+\lambda_{1}-\mu_{1}}$. The unique solution to $(4.3)$ in $(0, \infty) \times \mathcal{M}$ is

$$
u(x, 1)=x, \quad u(x, 2)=\frac{\lambda_{2}}{\lambda_{2}+r-\mu_{2}} x, \quad x>0 .
$$

Moreover, one can verify that $u(\cdot, \alpha), \alpha=1,2$ satisfy the subsolution property at the point $x=0$. Therefore $u$ is the unique constrained solution on $[0, \infty) \times\{1,2\}$.

The corresponding controlled dynamic is given by

$$
d X(t)=\mu_{\alpha(t)} X(t) d t+\sigma_{\alpha(t)} X(t) d W(t)-d Z(t),
$$

where $\alpha(t)$ is a two-state continuous-time Markov chain with generator $\left(\begin{array}{cc}-\lambda_{1} & \lambda_{1} \\ \lambda_{2} & -\lambda_{2}\end{array}\right)$. The objective is to maximize the reward functional $J(x, \alpha, Z)=$ $\mathbb{E}_{x, \alpha}\left[\int_{0}^{\infty} e^{-r t} d Z(t)\right]$. Observe that $\mathcal{A}_{x, \alpha} \neq \emptyset$ for all $(x, \alpha) \in[0, \infty) \times\{1,2\}$. Moreover, as demonstrated in Song et al. (2011), the value function $V(x, \alpha)=u(x, \alpha)$ for all $(x, \alpha)$, where $u$ is defined in (4.4).

Now let's present the main result of this section. 
Theorem 8 Assume $\mathcal{A}_{x, \alpha} \neq \emptyset$ and that the value function $V(\cdot, \alpha)$ defined in (2.1) is finite for each $(x, \alpha) \in$ $\bar{S} \times \mathcal{M}$. Then $\mathbf{V}(x)=(V(x, 1), \ldots, V(x, m))^{\prime}$ is a constrained viscosity solution of $(2.2)$ on $\bar{S} \times \mathcal{M}$.

The proof of Theorem 8 is similar to that of Song et al. (2011) with some modifications which are necessary to deal with the possibly discontinuous value function. In leu of the usual DPP, the proof utilizes the weak DPP Proposition 2. In addition, Proposition 3 is used to overcome certain technical issues. For brevity, we shall omit the details here.

\section{$5 \quad$ Viscosity Solution: Uniqueness}

Our goal is to establish a strong comparison result for constrained viscosity solutions of (2.2).

Lemma 9 Let $s(x)=x \cdot \mathbb{1}=\sum_{i=1}^{n} x_{i}$ and

$$
\tilde{u}(x, \alpha):=e^{-\lambda s(x)} u(x, \alpha), \tilde{v}(x, \alpha):=e^{-\lambda s(x)} v(x, \alpha),
$$

for all $(x, \alpha) \in S \times \mathcal{M}$, where $\lambda>0$. Then

(a) $u(x, \alpha)$ is viscosity subsolution of (2.2) if and only if $\tilde{u}(x, \alpha)$ is a viscosity subsolution of

$$
\begin{aligned}
& 0=\min \{r \tilde{u}(x, \alpha)-Q \tilde{u}(x, \cdot)(\alpha) \\
& -H_{\lambda}\left(x, \alpha, \tilde{u}(x, \alpha), D \tilde{u}(x, \alpha), D^{2} \tilde{u}(x, \alpha)\right), \\
& \left.\min _{i=1, \ldots, n}\left\{e^{\lambda s(x)}\left[\lambda \tilde{u}(x, \alpha)+D_{x_{i}} \tilde{u}(x, \alpha)\right]-f_{i}(x, \alpha)\right\}\right\},
\end{aligned}
$$

where for any $(x, \alpha, q, p, A) \in \mathbb{R}^{n} \times \mathcal{M} \times \mathbb{R} \times \mathbb{R}^{n} \times \mathcal{S}_{n}$,

$$
\begin{aligned}
& H_{\lambda}(x, \alpha, q, p, A)=\frac{1}{2} \operatorname{tr}\left(\sigma \sigma^{\prime}(x, \alpha) A\right) \\
& +\frac{\lambda}{2}\left(\mathbb{1}^{\prime} \sigma \sigma^{\prime}(x, \alpha) p+p^{\prime} \sigma \sigma^{\prime}(x, \alpha) \mathbb{1}\right) \\
& +b(x, \alpha)^{\prime} p+\lambda q b(x, \alpha)^{\prime} \mathbb{1}+\frac{\lambda^{2}}{2} q\left|\sigma^{\prime}(x, \alpha) \mathbb{1}\right|^{2},
\end{aligned}
$$

and $Q \tilde{u}(x, \cdot)(\alpha)=\sum_{j=1}^{m} q_{\alpha j}[\tilde{u}(x, j)-\tilde{u}(x, \alpha)]$.

(b) Similarly, $v(x, \alpha)$ is viscosity supersolution of (2.2) if and only if $\tilde{v}(x, \alpha)$ is a viscosity supersolution of $(5.2)$.

Proof. The proof follows directly from Definition 4 .

Lemma 10 For every $\xi \in \bar{S}$, there exist $\eta=\eta(\xi) \in \mathbb{R}^{n}$ and $a=a(\xi)>0$ such that

$$
B_{t a}(x+t \eta) \subset S, \quad \forall x \in \bar{S} \cap B_{a}(\xi), \forall t \in(0,1],
$$

where $B_{a}(x)=\left\{y \in \mathbb{R}^{n}:|y-x|<a\right\}$.
Proof. See Atar and Budhiraja (2006).

With Lemmas 9 and 10 at our hands, we are now ready to establish the strong comparison result.

Theorem 11 Let $\mathbf{u} \in U S C\left(\bar{S} \times \mathcal{M} ; \mathbb{R}^{m}\right)$ and $\mathbf{v} \in$ $L S C\left(\bar{S} \times \mathcal{M} ; \mathbb{R}^{m}\right)$ be respectively viscosity subsolution on $\bar{S} \times \mathcal{M}$ and supersolution in $S \times \mathcal{M}$ of (2.2) and satisfy

$$
|u(x, \alpha)|+|v(x, \alpha)| \leq K\left(1+|x|^{p}\right), \quad \forall(x, \alpha) \in \bar{S} \times \mathcal{M},
$$

where $K$ and $p$ are positive constants. Assume that for some positive constant $\kappa_{0}$, we have

$$
\begin{gathered}
|b(x, \alpha)-b(y, \alpha)|+|\sigma(x, \alpha)-\sigma(y, \alpha)| \leq \kappa_{0}|x-y| \\
b(x, \alpha)^{\prime} \mathbb{1} \leq \kappa_{0} \text { and }\left|\sigma(x, \alpha)^{\prime} \mathbb{1}\right| \leq \kappa_{0},
\end{gathered}
$$

for all $(x, \alpha) \in \bar{S} \times \mathcal{M}$. Then we have

$$
u(x, \alpha) \leq v(x, \alpha), \quad \forall(x, \alpha) \in \bar{S} \times \mathcal{M}
$$

Proof. Suppose on the contrary that

$$
M:=\max _{\alpha \in \mathcal{M}} \sup _{x \in \bar{S}}[u(x, \alpha)-v(x, \alpha)]>0
$$

We will derive a contradiction in the following. Define $\tilde{u}$ and $\tilde{v}$ as in (5.1), where $\lambda>0$ is a constant to be determined later. Thanks to (5.3), $\tilde{u}$ and $\tilde{v}$ are uniformly bounded. Moreover, we have

$$
\lim _{|x| \rightarrow \infty, x \in \bar{S}}(|\tilde{u}(x, \alpha)|+|\tilde{v}(x, \alpha)|)=0, \forall \alpha \in \mathcal{M}
$$

Thus in view of (5.6) and the facts that $\mathcal{M}$ is finite and that $\tilde{u}-\tilde{v}$ is upper semicontinuous (USC), there exist some bounded set $O$ of $\bar{S}$ and $(\hat{x}, \ell) \in O \times \mathcal{M}$, such that

$$
\begin{aligned}
\tilde{M} & :=\max _{\alpha \in \mathcal{M}} \sup _{x \in \bar{S}}[\tilde{u}(x, \alpha)-\tilde{v}(x, \alpha)] \\
& =\max _{x \in O}[\tilde{u}(x, \ell)-\tilde{v}(x, \ell)]=\tilde{u}(\hat{x}, \ell)-\tilde{v}(\hat{x}, \ell)>0 .
\end{aligned}
$$

Let $\eta=\eta(\hat{x})$ be as in Lemma 10 . For any $\varepsilon, \delta \in(0,1)$ and $(x, y) \in O \times O$, define

$$
\begin{aligned}
& \Phi(x, y)=\Phi_{\varepsilon, \delta, \lambda}(x, y):=\tilde{u}(x, \ell)-\tilde{v}(y, \ell)-\phi(x, y), \\
& \phi(x, y):=\left|\frac{1}{\varepsilon}(y-x)-\delta \eta\right|^{2}+\delta|x-\hat{x}|^{2} .
\end{aligned}
$$

Note that $\Phi$ is USC and hence achieves its maximum $M=M_{\varepsilon, \delta, \lambda}$ on the compact set $\bar{O}^{2}$ at $(\tilde{x}, \tilde{y}):=$ $\left(x_{\varepsilon, \delta, \lambda}, y_{\varepsilon, \delta, \lambda}\right)$. By virtue of Lemma 10, $\hat{x}+\varepsilon \delta \eta \in S^{o}$. 
Also, since

$$
\begin{aligned}
\Phi(\tilde{x}, \tilde{y}) & =\tilde{u}(\tilde{x}, \ell)-\tilde{v}(\tilde{y}, \ell)-\left|\frac{\tilde{y}-\tilde{x}}{\varepsilon}-\delta \eta\right|^{2}-\delta|\tilde{x}-\hat{x}|^{2} \\
& \geq \Phi(\hat{x}, \hat{x}+\varepsilon \delta \eta)=\tilde{u}(\hat{x}, \ell)-\tilde{v}(\hat{x}+\varepsilon \delta \eta, \ell)
\end{aligned}
$$

we have

$$
\begin{aligned}
& \tilde{u}(\tilde{x}, \ell)-\tilde{v}(\tilde{y}, \ell)-\tilde{u}(\hat{x}, \ell)+\tilde{v}(\hat{x}+\varepsilon \delta \eta, \ell) \\
& \geq\left|\frac{1}{\varepsilon}(\tilde{y}-\tilde{x})-\delta \eta\right|^{2}+\delta|\tilde{x}-\hat{x}|^{2} .
\end{aligned}
$$

Multiplying $\varepsilon^{2}$ on both sides of the above equation, we see that for each $\delta$ and $\lambda, \tilde{x}-\tilde{y} \rightarrow 0$ as $\varepsilon \rightarrow 0$. Further, by virtue of (5.7), we have $\lim \sup _{\varepsilon \rightarrow 0}\left|\frac{1}{\varepsilon}(\tilde{y}-\tilde{x})-\delta \eta\right|^{2}+$ $\delta|\tilde{x}-\hat{x}|^{2} \leq 0$; and therefore

$$
\tilde{x} \rightarrow \hat{x} \text {, and } \frac{1}{\varepsilon}(\tilde{y}-\tilde{x}) \rightarrow \delta \eta, \text { as } \varepsilon \rightarrow 0 .
$$

In particular, it follows that

$$
\tilde{y}=\tilde{x}+\varepsilon \delta \eta+o(\varepsilon)=\hat{x}+\varepsilon \delta \eta+o(\varepsilon), \text { as } \varepsilon \rightarrow 0,
$$

and hence $\tilde{y} \in S^{o}$ for $\varepsilon$ sufficiently small.

The function $x \mapsto \tilde{u}(x, \ell)-\phi_{1}(x)$ achieves its maximum at $\tilde{x}$, where $\phi_{1}(x)=\tilde{v}(\tilde{y}, \ell)+\left|\frac{1}{\varepsilon}(\tilde{y}-x)-\delta \eta\right|^{2}+$ $\delta|x-\hat{x}|^{2}$. Moreover, we compute

$$
\begin{aligned}
D \phi_{1}(x) & =-\frac{2}{\varepsilon}\left(\frac{1}{\varepsilon}(\tilde{y}-x)-\delta \eta\right)+2 \delta(x-\hat{x}), \text { and } \\
D^{2} \phi_{1}(x) & =\frac{2}{\varepsilon^{2}} I+2 \delta I .
\end{aligned}
$$

Hence it follows from Lemma 9, the definition of viscosity subsolution, and Ishii's lemma ((Crandall et al., 1992, Theorem 8.3)) that for some $M \in \mathcal{S}_{n},\left(-D \phi_{1}(\tilde{x}), M\right) \in$ $\overline{\mathcal{P}}^{2,+} \tilde{u}(\tilde{x}, \ell)$, such that

$$
\begin{aligned}
& 0 \geq \min \{ r \tilde{u}(\tilde{x}, \ell)-Q \tilde{u}(\tilde{x}, \cdot)(\ell) \\
&-H_{\lambda}\left(\tilde{x}, \ell, \tilde{u}(\tilde{x}, \ell), D \phi_{1}(\tilde{x}), M\right), \\
&\left.\min _{i=1, \ldots, n}\left\{e^{\lambda s(\tilde{x})}\left[\lambda \tilde{u}(\tilde{x}, \ell)+D \phi_{1}(\tilde{x}) \cdot e_{i}\right]-f_{i}(\tilde{x}, \ell)\right\}\right\} .
\end{aligned}
$$

Thus either

$$
\min _{i=1, \ldots, n}\left\{e^{\lambda s(\tilde{x})}\left[\lambda \tilde{u}(\tilde{x}, \ell)+D \phi_{1}(\tilde{x}) \cdot e_{i}\right]-f_{i}(\tilde{x}, \ell)\right\} \leq 0,
$$

or

$$
\begin{aligned}
& r \tilde{u}(\tilde{x}, \ell)-Q \tilde{u}(\tilde{x}, \cdot)(\ell) \\
& \quad-H_{\lambda}\left(\tilde{x}, \ell, \tilde{u}(\tilde{x}, \ell), D \phi_{1}(\tilde{x}), M\right) \leq 0 .
\end{aligned}
$$

On the other hand, the function $y \mapsto \tilde{v}(y, \ell)-\phi_{2}(y)$ achieves its minimum at $\tilde{y}$, where

$$
\phi_{2}(y)=\tilde{u}(\tilde{x}, \ell)-\left(\left|\frac{1}{\varepsilon}(y-\tilde{x})-\delta \eta\right|^{2}+\delta|\tilde{x}-\hat{x}|^{2}\right) .
$$

We compute $D \phi_{2}(y)=-\frac{2}{\varepsilon}\left(\frac{1}{\varepsilon}(y-\tilde{x})-\delta \eta\right)$, and $D^{2} \phi_{2}\left(y_{\varepsilon}\right)=-\frac{2}{\varepsilon^{2}} I$. Hence the definition of supersolution and Ishii's lemma ((Crandall et al., 1992, Theorem 8.3)) imply that for some $N \in \mathcal{S}_{n}$, we have $\left(D \phi_{2}(\tilde{y}), N\right) \in \overline{\mathcal{P}}^{2,-} \tilde{v}(\tilde{y}, \ell)$ and

$$
\begin{aligned}
& 0 \leq \min \{r \tilde{v}(\tilde{y}, \ell)-Q \tilde{v}(\tilde{y}, \cdot)(\ell) \\
&-H_{\lambda}\left(\tilde{y}, \ell, \tilde{v}(\tilde{y}, \ell), D \phi_{2}(\tilde{y}), N\right) \\
&\left.\min _{i=1, \ldots, n}\left\{e^{\lambda s(\tilde{y})}\left[\lambda \tilde{v}(\tilde{y}, \ell)+D \phi_{2}(\tilde{y}) \cdot e_{i}\right]-f_{i}(\tilde{y}, \ell)\right\}\right\} .
\end{aligned}
$$

Case 1. Now suppose (5.11) is true. Recall $\tilde{u}(x, \alpha)=$ $e^{-\lambda s(x)} u(x, \alpha)$ and $\tilde{v}(x, \alpha)=e^{-\lambda s(x)} v(x, \alpha)$. Then we have from (5.11) and (5.13) that

$$
\begin{aligned}
& \min _{i=1, \ldots, n}\left\{\lambda(u(\tilde{x}, \ell)-v(\tilde{x}, \ell))-\left(f_{i}(\tilde{x}, \ell)-f_{i}(\tilde{y}, \ell)\right)\right. \\
& \left.+\left(e^{\lambda s(\tilde{x})} D \phi_{1}(\tilde{x})-e^{\lambda s(\tilde{y})} D \phi_{2}(\tilde{y})\right) \cdot e_{i}\right\} \leq 0 .
\end{aligned}
$$

Hence it follows that

$$
\begin{aligned}
& \lambda(u(\tilde{x}, \ell)-v(\tilde{x}, \ell)) \leq \max _{i=1, \ldots, n}\left\{\left(f_{i}(\tilde{x}, \ell)-f_{i}(\tilde{y}, \ell)\right)\right. \\
& \left.-\left(e^{\lambda s(\tilde{x})} D \phi_{1}(\tilde{x})-e^{\lambda s(\tilde{y})} D \phi_{2}(\tilde{y})\right) \cdot e_{i}\right\} \\
& =\max _{i=1, \ldots, n}\left\{\left(f_{i}(\tilde{x}, \ell)-f_{i}(\tilde{y}, \ell)\right)\right. \\
& -e_{i} \cdot\left[e^{\lambda s(\tilde{x})}\left(-\frac{2}{\varepsilon}\left(\frac{1}{\varepsilon}(\tilde{y}-\tilde{x})-\delta \eta\right)+2 \delta(\tilde{x}-\hat{x})\right)\right. \\
& \left.\left.+e^{\lambda s(\tilde{y})} \frac{2}{\varepsilon}\left(\frac{1}{\varepsilon}(\tilde{y}-\tilde{x})-\delta \eta\right)\right]\right\} .
\end{aligned}
$$

Thanks to (5.9), (5.10), and the continuity of $f_{i}$, the right-hand-side of (5.14) converges to 0 as $\varepsilon \downarrow 0$. Note that for any $x \in O$, we have

$$
\begin{aligned}
& \tilde{u}(x, \ell)-\tilde{v}(x, \ell)=\Phi(x, x) \leq \Phi(\tilde{x}, \tilde{y}) \\
& \quad=\tilde{u}(\tilde{x}, \ell)-\tilde{v}(\tilde{y}, \ell)-\phi(\tilde{x}, \tilde{y}) \leq \tilde{u}(\tilde{x}, \ell)-\tilde{v}(\tilde{y}, \ell) .
\end{aligned}
$$

In particular, taking $x=\hat{x}$ leads to $\tilde{u}(\hat{x}, \ell)-\tilde{v}(\hat{x}, \ell) \leq 0$, which gives a contradiction to (5.7). Hence, Case 1 is impossible.

Case 2. Now suppose (5.12) is true. Then it follows from 
(5.12) and (5.13) that

$$
\begin{aligned}
& r(\tilde{u}(\tilde{x}, \ell)-\tilde{v}(\tilde{y}, \ell))-[Q \tilde{u}(\tilde{x}, \cdot)(\ell)-Q \tilde{v}(\tilde{y}, \cdot)(\ell)] \\
& -\left[H_{\lambda}\left(\tilde{x}, \ell, \tilde{u}(\tilde{x}, \ell), D \phi_{1}(\tilde{x}), M\right)\right. \\
& \left.\quad-H_{\lambda}\left(\tilde{y}, \ell, \tilde{v}\left(y_{\varepsilon}, \ell\right), D \phi_{2}(\tilde{y}), N\right)\right] \leq 0
\end{aligned}
$$

Using the definition of $H_{\lambda}$,

$$
\begin{aligned}
& H_{\lambda}\left(\tilde{x}, \ell, \tilde{u}(\tilde{x}, \ell), D \phi_{1}(\tilde{x}), M\right) \\
& \quad-H_{\lambda}\left(\tilde{y}, \ell, \tilde{v}(\tilde{y}, \ell), D \phi_{2}(\tilde{y}), N\right) \\
& =\frac{1}{2}\left(\operatorname{tr}\left(\sigma \sigma^{\prime}(\tilde{x}, \ell) M\right)-\operatorname{tr}\left(\sigma \sigma^{\prime}(\tilde{y}, \ell) N\right)\right) \\
& \quad+\lambda(\tilde{u}(\tilde{x}, \ell) b(\tilde{x}, \ell)-\tilde{v}(\tilde{y}, \ell) b(\tilde{y}, \ell)) \cdot \mathbb{1} \\
& \quad+\frac{\lambda}{2}\left(\left[\mathbb{1}^{\prime} \sigma \sigma^{\prime}(\tilde{x}, \ell) D \phi_{1}(\tilde{x})+D \phi_{1}(\tilde{x})^{\prime} \sigma \sigma^{\prime}(\tilde{x}, \ell) \mathbb{1}\right]\right. \\
& \left.\quad-\left[\mathbb{1}^{\prime} \sigma \sigma^{\prime}(\tilde{y}, \ell) D \phi_{2}(\tilde{y})+D \phi_{2}(\tilde{y})^{\prime} \sigma \sigma^{\prime}(\tilde{y}, \ell) \mathbb{1}\right]\right) \\
& \quad+b(\tilde{x}, \ell) \cdot D \phi_{1}(\tilde{x})-b(\tilde{y}, \ell) \cdot D \phi_{2}(\tilde{y}) \\
& \quad+\frac{\lambda^{2}}{2}\left(\tilde{u}(\tilde{x}, \ell)\left|\sigma^{\prime}(\tilde{x}, \ell) \mathbb{1}\right|^{2}-\tilde{v}(\tilde{y}, \ell)\left|\sigma^{\prime}(\tilde{y}, \ell) \mathbb{1}\right|^{2}\right) .
\end{aligned}
$$

Hence it follows that

$$
\begin{aligned}
{[r} & \left.-\lambda b(\tilde{x}, \ell)^{\prime} \mathbb{1}-\frac{\lambda^{2}}{2}\left|\sigma^{\prime}(\tilde{x}, \ell) \mathbb{1}\right|^{2}\right](\tilde{u}(\tilde{x}, \ell)-\tilde{v}(\tilde{y}, \ell)) \\
\leq & {[Q \tilde{u}(\tilde{x}, \cdot)(\ell)-Q \tilde{v}(\tilde{y}, \cdot)(\ell)] } \\
& +\lambda \tilde{v}(\tilde{y}, \ell)(b(\tilde{x}, \ell)-b(\tilde{y}, \ell)) \cdot \mathbb{1} \\
& +\frac{1}{2}\left[\operatorname{tr}\left(\sigma \sigma^{\prime}(\tilde{x}, \ell) M\right)-\operatorname{tr}\left(\sigma \sigma^{\prime}(\tilde{y}, \ell) N\right)\right] \\
& +\frac{\lambda}{2}\left(\left[\mathbb{1}^{\prime} \sigma \sigma^{\prime}(\tilde{x}, \ell) D \phi_{1}(\tilde{x})+D \phi_{1}(\tilde{x})^{\prime} \sigma \sigma^{\prime}(\tilde{x}, \ell) \mathbb{1}\right]\right. \\
& \left.-\left[\mathbb{1}^{\prime} \sigma \sigma^{\prime}(\tilde{y}, \ell) D \phi_{2}(\tilde{y})+D \phi_{2}(\tilde{y})^{\prime} \sigma \sigma^{\prime}(\tilde{y}, \ell) \mathbb{1}\right]\right) \\
& +b(\tilde{x}, \ell) \cdot D \phi_{1}(\tilde{x})-b(\tilde{y}, \ell) \cdot D \phi_{2}(\tilde{y}) \\
& +\frac{\lambda^{2}}{2} \tilde{v}(\tilde{y}, \ell)\left(\left|\sigma^{\prime}(\tilde{x}, \ell) \mathbb{1}\right|^{2}-\left|\sigma^{\prime}(\tilde{y}, \ell) \mathbb{1}\right|^{2}\right) .
\end{aligned}
$$

Using (5.5) and the fact that $r>0$, we choose some $\lambda>0$ sufficiently small so that

$$
r-\lambda b(\tilde{x}, \ell)^{\prime} \mathbb{1}-\frac{\lambda^{2}}{2}\left|\sigma^{\prime}(\tilde{x}, \ell) \mathbb{1}\right|^{2}>0 .
$$

Next we analyze the terms on the right-hand side of (5.16). By virtue of Ishii's lemma,

$$
\lim _{\varepsilon \downarrow 0} \frac{1}{2}\left(\operatorname{tr}\left(\sigma \sigma^{\prime}\left(x_{\varepsilon}, \ell\right) M\right)-\operatorname{tr}\left(\sigma \sigma^{\prime}\left(y_{\varepsilon}, \ell\right) N\right)\right)=0 .
$$

Recall that

$$
\begin{aligned}
\Phi(\tilde{x}, \tilde{y}) & =\tilde{u}(\tilde{x}, \ell)-\tilde{v}(\tilde{y}, \ell)-\phi(\tilde{x}, \tilde{y}) \\
& \geq \Phi(\hat{x}, \hat{x})=\tilde{u}(\hat{x}, \ell)-\tilde{v}(\hat{x}, \ell)-\delta^{2}|\eta|^{2} .
\end{aligned}
$$

Note also $q_{\ell \ell}<0$. Thus it follows that

$$
\begin{aligned}
& q_{\ell \ell} {[\tilde{u}(\tilde{x}, \ell)-\tilde{v}(\tilde{y}, \ell)] } \\
& \quad \leq q_{\ell \ell}\left[\tilde{u}(\hat{x}, \ell)-\tilde{v}(\hat{x}, \ell)+\phi(\tilde{x}, \tilde{y})-\delta^{2}|\eta|^{2}\right] .
\end{aligned}
$$

This, together with (5.7), (5.9), (5.10), and the fact that $\tilde{u}-\tilde{v}$ is USC, lead to

$$
\begin{aligned}
& \limsup _{\varepsilon \downarrow 0}[Q \tilde{u}(\tilde{x}, \cdot)(\ell)-Q \tilde{v}(\tilde{y}, \cdot)(\ell)] \\
& =\limsup _{\varepsilon \downarrow 0} \sum_{j \neq \ell} q_{\ell j}(\tilde{u}(\tilde{x}, j)-\tilde{v}(\tilde{y}, j)) \\
& \quad+q_{\ell \ell \ell}(\tilde{u}(\tilde{x}, \ell)-\tilde{v}(\tilde{y}, \ell)) \\
& \leq \sum_{j \neq \ell} q_{\ell j}(\tilde{u}(\hat{x}, j)-\tilde{v}(\hat{x}, j))-\delta^{2} q_{\ell \ell}|\eta|^{2} \\
& \quad+q_{\ell \ell}(\tilde{u}(\hat{x}, \ell)-\tilde{v}(\hat{x}, \ell)) \\
& \leq \sum_{j \neq \ell} q_{\ell j}(\tilde{u}(\hat{x}, \ell)-\tilde{v}(\hat{x}, \ell))-\delta^{2} q_{\ell \ell}|\eta|^{2} \\
& \quad+q_{\ell \ell}(\tilde{u}(\hat{x}, \ell)-\tilde{v}(\hat{x}, \ell))=-\delta^{2} q_{\ell \ell}|\eta|^{2} .
\end{aligned}
$$

Next, using (5.4) and (5.9), and noting that $\tilde{v}$ is bounded, we have

$$
\begin{aligned}
& \lim _{\varepsilon \downarrow 0}[\lambda \tilde{v}(\tilde{y}, \ell)(b(\tilde{x}, \ell)-b(\tilde{y}, \ell)) \cdot \mathbb{1} \\
& \left.+\frac{\lambda^{2}}{2} \tilde{v}(\tilde{y}, \ell)\left(\left|\sigma^{\prime}(\tilde{x}, \ell) \mathbb{1}\right|^{2}-\left|\sigma^{\prime}(\tilde{y}, \ell) \mathbb{1}\right|^{2}\right)\right]=0 .
\end{aligned}
$$

Similarly (5.4) and (5.9) imply that

$$
\begin{aligned}
& {\left[\mathbb{1}^{\prime} \sigma \sigma^{\prime}(\tilde{x}, \ell) D \phi_{1}(\tilde{x})+D \phi_{1}(\tilde{x})^{\prime} \sigma \sigma^{\prime}(\tilde{x}, \ell) \mathbb{1}\right]} \\
& \quad-\left[\mathbb{1}^{\prime} \sigma \sigma^{\prime}(\tilde{y}, \ell) D \phi_{2}(\tilde{y})+D \phi_{2}(\tilde{y})^{\prime} \sigma \sigma^{\prime}(\tilde{y}, \ell) \mathbb{1}\right] \\
& =-\frac{2}{\varepsilon} \mathbb{1}^{\prime}\left(\sigma \sigma^{\prime}(\tilde{x}, \ell)-\sigma \sigma^{\prime}(\tilde{y}, \ell)\right) \cdot\left(\frac{1}{\varepsilon}(\tilde{y}-\tilde{x})-\delta \eta\right) \\
& \quad-\frac{2}{\varepsilon}\left(\frac{1}{\varepsilon}(\tilde{y}-\tilde{x})-\delta \eta\right)^{\prime}\left(\sigma \sigma^{\prime}(\tilde{x}, \ell)-\sigma \sigma^{\prime}(\tilde{y}, \ell)\right) \mathbb{1} \\
& \quad+2 \delta\left(\mathbb{1}^{\prime} \sigma \sigma^{\prime}(\tilde{x}, \ell)(\tilde{x}-\hat{x})+(\tilde{x}-\hat{x})^{\prime} \sigma \sigma^{\prime}(\tilde{x}, \ell) \mathbb{1}\right) \\
& \rightarrow 0, \text { as } \varepsilon \rightarrow 0 ;
\end{aligned}
$$

and

$$
\begin{aligned}
& b(\tilde{x}, \ell) \cdot D \phi_{1}(\tilde{x})-b(\tilde{y}, \ell) D \phi_{2}(\tilde{y}) \\
& =-\frac{2}{\varepsilon}(b(\tilde{x}, \ell)-b(\tilde{y}, \ell)) \cdot\left(\frac{1}{\varepsilon}(\tilde{y}-\tilde{x})-\delta \eta\right) \\
& \quad+2 \delta b(\tilde{x}, \ell) \cdot(\tilde{x}-\hat{x}) \\
& \rightarrow 0, \quad \text { as } \varepsilon \rightarrow 0 .
\end{aligned}
$$

Now letting $\varepsilon \downarrow 0$ and using (5.17)-(5.22) in (5.16), it follows that

$$
\limsup _{\varepsilon \rightarrow 0} \tilde{u}(\tilde{x}, \ell)-\tilde{v}(\tilde{y}, \ell) \leq-\delta^{2} q_{\ell \ell}|\eta|^{2} .
$$


But $\delta>0$ can be arbitrarily small, as argued in Case 1, it follows that

$$
\tilde{u}(\hat{x}, \ell)-\tilde{v}(\hat{x}, \ell) \leq \tilde{u}\left(x_{\varepsilon}, \ell\right)-\tilde{v}\left(y_{\varepsilon}, \ell\right) \rightarrow 0
$$

as $\varepsilon \rightarrow 0$ and $\delta \rightarrow 0$, which again contradicts (5.7). Therefore for any $x \in S$ and $\alpha \in \mathcal{M}$, we have $u(x, \alpha) \leq$ $v(x, \alpha)$, as desired.

Remark 12 Note that under condition (5.5), the value function is bounded above by an affine function. In fact, for any $(x, \alpha) \in S \times \mathcal{M}$ and $Z \in \mathcal{A}_{x, \alpha}$, we have

$$
\begin{aligned}
d\left(e^{-r t} X(t)\right)= & e^{-r t}[(b(X(t), \alpha(t))-r X(t)) d t \\
& +\sigma(X(t), \alpha(t)) d W(t)-d Z(t)] .
\end{aligned}
$$

Thus

$$
\begin{gathered}
e^{-r t} d Z(t)=e^{-r t}(b(X(t), \alpha(t))-r X(t)) d t \\
+e^{-r t} \sigma(X(t), \alpha(t)) d W(t)-d\left(e^{-r t} X(t)\right),
\end{gathered}
$$

from which it follows that

$$
\begin{array}{r}
\int_{0}^{\infty} e^{-r t} \mathbb{1} \cdot d Z(t) \leq \int_{0}^{\infty} e^{-r t} \mathbb{1} \cdot[(b(X(t), \alpha(t)) \\
-r X(t)) d t+\sigma(X(t), \alpha(t)) d W(t)]+\mathbb{1} \cdot x
\end{array}
$$

Taking expectations on both sides and using (5.5), we have

$$
\begin{aligned}
& \mathbb{E}\left[\int_{0}^{\infty} e^{-r t} \mathbb{1} \cdot d Z(t)\right] \leq \mathbb{1} \cdot x \\
& +\mathbb{E}\left[\int_{0}^{\infty} e^{-r t}\left(\kappa_{0} d t+\mathbb{1} \cdot \sigma(X(t), \alpha(t)) d W(t)\right)\right] \\
& =\frac{\kappa_{0}}{r}+\mathbb{1} \cdot x .
\end{aligned}
$$

Hence, it follows that

$$
\begin{aligned}
V(x, \alpha) & =\sup _{Z \in \mathcal{A}_{x, \alpha}} \mathbb{E}\left[\int_{0}^{\infty} e^{-r t} f(X(t-), \alpha(t-) \cdot d Z(t)]\right. \\
& \leq\|f\|_{\infty}\left(\frac{\kappa_{0}}{r}+\mathbb{1} \cdot x\right) .
\end{aligned}
$$

Finally we summarize the main result of this paper from Theorems 8 and 11 .

Theorem 13 Assume (5.4), (5.5), and that $\mathcal{A}_{x, \alpha} \neq \emptyset$ for every $(x, \alpha) \in S \times \mathcal{M}$. Then the value function $V$ defined in (2.1) is the unique constrained viscosity solution of the system of coupled quasi-variational inequalities (2.2) on $\bar{S} \times \mathcal{M}$.

Remark 14 At first look, condition (5.5) seems rather restrictive. However, the following example indicates that in general, one can not remove (5.5); otherwise, uniqueness may not hold.
Example 15 Let's consider a 1-dimensional squared Bessel process subject to control

$$
d X(t)=d t+2 \sqrt{|X(t)|} d W(t)-d Z(t)
$$

with reward functional $J(x, Z)=\mathbb{E}_{x}\left[\int_{0}^{\infty} e^{-t} d Z(t)\right]$, where $x>0$. It is well known (see, e.g., Revuz and Yor (1999)) that $\xi^{x}(t)=x+|W(t)|^{2} \geq 0$ if $x \geq 0$. Moreover, we have $\mathbb{P}_{0}\{\tau=0\}=1$, where $\tau:=\inf \left\{t>0: \xi^{0}(t)>0\right\}$. Hence it follows that $\mathcal{A}_{x} \neq \emptyset$ for all $x \in[0, \infty)$.

The corresponding QVI is

$$
\min \left\{u(x)-u^{\prime}(x)-2 x u^{\prime \prime}(x), u^{\prime}(x)-1\right\}=0, x>0 .
$$

One can directly verify that $v(x)=x+1$ is a constrained viscosity solution to $(5.24)$ on $[0, \infty)$.

Next, we demonstrate that (5.24) has at least another constrained viscosity solution on $[0, \infty)$. First we note that the function $\psi(x):=\sinh (\sqrt{2 x})$ is increasing and solves the equation $u(x)-u^{\prime}(x)-2 x u^{\prime \prime}(x)=0$ in $(0, \infty)$. Further, straightforward calculations reveal that

$$
\begin{aligned}
\psi^{\prime}(x) & =\frac{\cosh (\sqrt{2 x})}{\sqrt{2 x}}, \\
\psi^{\prime \prime}(x) & =\frac{1}{2 \sqrt{2}}\left[-\frac{\cosh (\sqrt{2 x})}{x^{3 / 2}}+\frac{\sqrt{2} \sinh (\sqrt{2 x})}{x}\right] .
\end{aligned}
$$

The equation $\psi^{\prime \prime}(x)=0$ or equivalently $\frac{\cosh (\sqrt{2 x})}{\sinh (\sqrt{2 x})}=\sqrt{2 x}$ has a unique positive root, denoted by $z$. Now we claim that the function defined by

$$
\begin{aligned}
u(x) & =\sinh (\sqrt{2 x}) \frac{\sqrt{2 z}}{\cosh (\sqrt{2 z})} I_{[0, z]}(x) \\
& +\left(x-z+\sinh (\sqrt{2 z}) \frac{\sqrt{2 z}}{\cosh (\sqrt{2 z})}\right) I_{(z, \infty)}(x)
\end{aligned}
$$

is another constrained viscosity solution to (5.24) on $[0, \infty)$. In fact, one can directly verify that $u(x)$ is a solution to (5.24) for $x>0$. It remains to verify the subsolution property at the point $x=0$. To this end, let $\phi \in C^{2}$ with $(u-\phi)(x) \leq(u-\phi)(0)=0$ for $x \in[0, \infty)$ in a neighborhood of 0 . Then $\phi(0)=0$ and for $x>0$, $\phi(x) \geq u(x)>0$. Thus we must have $\phi^{\prime}(0) \geq 0$ and hence

$$
\min \left\{\phi(0)-\phi^{\prime}(0)-0 \cdot \phi^{\prime \prime}(0), \phi^{\prime}(0)-1\right\} \leq 0 .
$$

The desired conclusion follows. Note that $u$ also satisfies the polynomial growth condition (5.3).

In terms of the singular control problem (5.23), it turns out that the value function $V(x)=v(x)=x+1$ with the optimal control $Z^{*}(t)=x+W^{2}(t)$. 


\section{Conclusions and Remarks}

In this work, we considered a class of singular control problems with state constraints and regime-switching. The controlled dynamic is given by a regime-switching diffusion confined in the unbounded domain $S=\mathbb{R}_{+}^{n}$ and the objective is to maximize the total expected discounted rewards from exerting the singular control. Using the weak dynamic programming principle and an appropriate exponential transformation, we showed that the value function is the unique constrained viscosity solution of the system of coupled nonlinear quasivariational inequalities $(2.2)$.

This paper is focused on the discounted criterion with an infinite horizon. It will be interesting to study the problem using the long-term average criterion. A number of other questions deserve further investigations. In particular, it is worth studying the case when the random environment or the Markov chain $\alpha$ is unobservable.

\section{References}

Alvarez, L. H. R. (2000). Singular stochastic control in the presence of a state-dependent yield structure. Stochastic Process. Appl., 86(2):323-343.

Alvarez, L. H. R. and Shepp, L. A. (1998). Optimal harvesting of stochastically fluctuating populations. J. Math. Biol., 37(2):155-177.

Atar, R. and Budhiraja, A. (2006). Singular control with state constraints on unbounded domain. Ann. Probab., 34(5):1864-1909.

Atar, R., Budhiraja, A., and Williams, R. J. (2007). HJB equations for certain singularly controlled diffusions. Ann. Appl. Probab., 17(5-6):1745-1776.

Bouchard, B. and Touzi, N. (2011). Weak dynamic programming principle for viscosity solutions. SIAM Journal on Control and Optimization, 49(3):948-962.

Crandall, M. G., Ishii, H., and Lions, P.-L. (1992). User's guide to viscosity solutions of second order partial differential equations. Bull. Amer. Math. Soc. (N.S.), 27(1):1-67.

Elliott, R. J. and Siu, T. K. (2010). On risk minimizing portfolios under a Markovian regime-switching BlackScholes economy. Ann. Oper. Res., 176:271-291.

Guo, X. and Pham, H. (2005). Optimal partially reversible investment with entry decision and general production function. Stochastic Process. Appl., 115(5):705-736.

Haussmann, U. G. and Suo, W. (1995a). Singular optimal stochastic controls. I. Existence. SIAM J. Control Optim., 33(3):916-936.

Haussmann, U. G. and Suo, W. (1995b). Singular optimal stochastic controls. II. Dynamic programming. SIAM J. Control Optim., 33(3):937-959.

Ishii, H. (1989). On uniqueness and existence of viscosity solutions of fully nonlinear second-order elliptic PDEs. Comm. Pure Appl. Math., 42(1):15-45.
Karatzas, I. and Shreve, S. E. (1984). Connections between optimal stopping and singular stochastic control. I. Monotone follower problems. SIAM J. Control Optim., 22(6):856-877.

Karlin, S. and Taylor, H. M. (1981). A second course in stochastic processes. Academic Press Inc. [Harcourt Brace Jovanovich Publishers], New York.

Lee, C. and Weerasinghe, A. (2011). Convergence of a queueing system in heavy traffic with general patience-time distributions. Stochastic Process. Appl., 121(11):2507-2552.

Mao, X. and Yuan, C. (2006). Stochastic differential equations with Markovian switching. Imperial College Press, London.

Øksendal, B. and Sulem, A. (2002). Optimal consumption and portfolio with both fixed and proportional transaction costs. SIAM J. Control Optim., 40(6):1765-1790.

Paulsen, J. (2003). Optimal dividend payouts for diffusions with solvency constraints. Finance Stoch., $7(4): 457-473$.

Revuz, D. and Yor, M. (1999). Continuous martingales and Brownian motion, volume 293 of Grundlehren der Mathematischen Wissenschaften [Fundamental Principles of Mathematical Sciences]. Springer-Verlag, Berlin, third edition.

Sethi, S. P. and Zhang, Q. (1994). Hierarchical decision making in stochastic manufacturing systems. Systems \& Control: Foundations \& Applications. Birkhäuser Boston Inc., Boston, MA.

Song, Q. S., Stockbridge, R. H., and Zhu, C. (2011). On optimal harvesting problems in random environments. SIAM J. Control Optim., 49(2):859-889.

Yin, G. G. and Zhu, C. (2010). Hybrid Switching Diffusions: Properties and Applications, volume 63 of Stochastic Modelling and Applied Probability. Springer, New York.

Yong, J. and Zhou, X. Y. (1999). Stochastic Controls: Hamiltonian Systems and HJB Equations, volume 43 of Applications of Mathematics (New York). SpringerVerlag, New York.

Zariphopoulou, T. (1992). Investment-consumption models with transaction fees and Markov-chain parameters. SIAM J. Control Optim., 30(3):613-636.

Zhang, Q. (2001). Stock trading: an optimal selling rule. SIAM J. Control Optim., 40(1):64-87.

Zhu, C. (2011). Optimal control of risk process in a regime-switching environment. Automatica, 47(8):1570-1579.

Zhu, C. and Yin, G. (2009). On competitive LotkaVolterra model in random environments. J. Math. Anal. Appl., 357(1):154-170. 